\section{Cognitive impairment in neuromuscular diseases: A systematic review}

\author{
Marco Orsini, 1,2 Ana Carolina \\ Andorinho de F. Ferreira, ${ }^{3}$ \\ Anna Carolina Damm de Assis, 3,4 \\ Thais Magalhães, ${ }^{3}$ Silmar Teixeira, ${ }^{2}$ \\ Victor Hugo Bastos, ${ }^{2}$ Victor Marinho, ${ }^{2}$ \\ Thomaz Oliveira, ${ }^{2}$ Rossano Fiorelli, ${ }^{1}$ \\ Acary Bulle Oliveira, 4 \\ Marcos R.G. de Freitas ${ }^{5}$ \\ 1Master's Program in Health Applied \\ Sciences, Severino Sombra University, \\ Vasssouras, Rio de Janeiro; ${ }^{2}$ Brain \\ Mapping and Plasticity Laboratory, \\ Federal University of Piauí, Parnaíba; \\ 3Department of Neurology, Federal \\ Fluminense University, Rio de Janeiro; \\ ${ }^{4}$ Department of Neurology, Federal \\ University of São Paulo; 5Department of \\ Neurology, Federal University of Rio de \\ Janeiro, Brazil
}

\begin{abstract}
Neuromuscular diseases are multifactorial pathologies characterized by extensive muscle fiber damage that leads to the activation of satellite cells and to the exhaustion of their pool, with consequent impairment of neurobiological aspects, such as cognition and motor control. To review the knowledge and obtain a broad view of the cognitive impairment on Neuromuscular Diseases. Cognitive impairment in neuromuscular disease was explored; a literature search up to October 2017 was conducted, including experimental studies, case reports and reviews written in English. Keywords included Cognitive Impairment, Neuromuscular Diseases, Motor Neuron Diseases, Dystrophinopathies and Mitochondrial Disorders. Several cognitive evaluation scales, neuroimaging scans, genetic analysis and laboratory applications in neuromuscular diseases, especially when it comes to the Motor Neuron Diseases, Dystrophinopathies and Mitochondrial Disorders. In addition, organisms model using rats in the genetic analysis and laboratory applications to verify the cognitive and neuromuscular impacts. Several studies indicate that congenital molecular alterations in neuromuscular diseases promote cognitive dysfunctions. Understanding these mechanisms may in the future guide the proper management of the patient, evaluation, establishment of prognosis, choice of treatment and development of innovative interventions such as gene therapy.
\end{abstract}

\section{Introduction}

Neuromuscular diseases present a wide variety of clinical manifestations, but their effects on the cognitive function spectrum are still poorly understood. ${ }^{1}$ In contrast to the studies on how altered executive functions in mental disorders such as anxiety, depression and bipolar disorder can affect motor performance, ${ }^{2-4}$ the mechanisms by which essentially motor dysfunctions can affect cognitive performance still remain poorly understood and studied.5-8 Although it is known that neuromuscular diseases mainly affect the motor functioning of the patient, the cognitive effects of these conditions can be significant. 5 This can occur from molecular defects that significantly affect neuromotor functioning but also participate in the functioning of neural networks involved in cognitive processes, leading to dysfunctions of executive, behavioral and psychosocial functions. $^{6-8}$ For example, mutations in the dystrophin protein gene, responsible for the Duchenne Muscular Dystrophy (DMD) phenotype, concurrently affect the stability and function of muscle fibers and central nervous system (CNS) function. However, the complex nature of neuromuscular diseases and the different effects on cognition make it difficult to understand the neuropathological process. $.7,8$

Three large, heterogeneous groups of neuromuscular diseases have been implicated in cognitive alterations. i) Motor Neuron Diseases, neurological disorders that affect the neurons that control voluntary motor activity;9,10 ii) Muscular dystrophies, represented mainly by dystrophinopathies, diseases caused by hereditary defects in the dystrophin protein gene;11 iii) Mitochondrial disorders, which are caused by mutations in cytoplasmic or nuclear mitochondrial DNA, which alter the structure and mitochondria function. ${ }^{10,12}$ Often neuromuscular disease comes from an already known molecular disorder that is also associated with changes in brain function and cognition, such as mitochondrial myopathies. In other cases, neuromuscular disorders also have cognitive deficits, but without a well-defined molecular alteration such as Amyotrophic Lateral Sclerosis (ALS). ${ }^{8,13}$ In this context, the present review aims to highlight the neurofunctional and cognitive alterations in the main congenital neuromuscular disorders.

\section{Materials and Methods}

The systematic review was conducted in line with the PRISMA statement. A com-
Correspondence: Francisco Victor Costa Marinho, Federal University of Piauí, Brazil. Brain Mapping and Plasticity Laboratory- Av. São Sebastião n²819 - Nossa Sra. de Fátima - Parnaíba, PI, CEP: 64202-020, Brazil.

Tel.: +55.86.994178117.

E-mail: victormarinhophb@hotmail.com

Key words: Cognitive Impairment; Neuromuscular Diseases; Motor Neuron Diseases; Dystrophinopathies; Mitochondrial Disorders.

Contributions: the authors contributed equally.

Conflict of interest: the authors declare no potential conflict of interest.

Funding: none.

Received for publication: 31 October 2017.

Revision received: 4 December 2017

Accepted for publication: 12 December 2017.

This work is licensed under a Creative Commons Attribution NonCommercial 4.0 License (CC BY-NC 4.0).

(C) Copyright M. Orsini et al., 2018

Licensee PAGEPress, Italy

Neurology International 2018; 10:7473

doi:10.4081/ni.2018.7473

puter-based literature search was conducted in two main databases: ISI Web of Science and PubMed (2003-present) were initially performed in October 2017 using relevant search terms: (e.g. [Cognitive Impairment and Neuromuscular Diseases] [Motor Neuron Diseases and Cognitive Impairment] [Cognitive Impairment and Dystrophinopathies] [Cognitive Impairment and Mitochondrial Disorders]. Abstracts were examined for references to the research question and if the study appeared relevant, then the full text was retrieved. As selection criteria: (a) Experimental studies, case reports and reviews written in English; (b) Without restriction of publication dates of the paper (c) Magnetic Resonance Imaging (MRI) scans, assessments with cognitive and functional scales applied to neuromuscular diseases, and cognitive tasks healthy individuals and/or individuals with neuromuscular diseases (e.g. children, young adults, middle-aged and elderly). In addition, experimental studies using rats were considered. Criteria for exclusion were: (a) dissertations, book reviews, conference proceedings, or editorials. Data were extracted based on study design and setting. The discussion was written after observing common points among the articles selected. This observation was conducted in order to 
analyze the papers results and answer the hypothesis of the present research.

\section{Study selection and data extraction}

Three reviewers (M.O, S.T and V.M) independently read the titles and/or abstracts of the identified papers and eliminated irrelevant studies. Studies considered eligible for inclusion were read in full and their suitability for inclusion was determined independently by three reviewers (M.O, S.T and V.M) Disagreements were managed by consensus. However, if this was not successful, consensus was sought by a fourth reviewer (M.R.G.F.). Data were extracted based on study design and setting. Some authors were contacted to provide supplementary information when insufficient data were provided in the study. The authors of three studies were contacted for further information having read their titles and abstracts. Two replied; since it bases the review of literature fulfilled the inclusion criteria.

\section{Study selection}

Studies were included if they met the following inclusion criteria. Initially, retrieved papers from each database were compared to remove duplicate records. Papers were then screened for eligibility based on their title and abstract and, once they were elicited, the full text publication was reviewed.

In summary: Study design: Case reports, original papers and reviews designs were included.

Population: Study population composed of healthy individuals and/or individuals with neuromuscular diseases (e.g., children, young adults, middle-aged and elderly). In addition, experimental studies with rats were included.

Intervention: Neurobiological interventions were defined as any intervention aimed at evaluating the neuromuscular diseases - Motor Neuron Diseases, Dystrophinopathies and Mitochondrial. Disorders.

Screening: Genetic analysis in vivo and post-mortem, MRI scans, mobile transcutaneous sensor for tissue blood gas tensions, ALS Functional Rating Scale, Reye Osterrieth Complex Figure Test, Premorbid Full-Scale IQ, Wechsler Test of Adult Reading, Pyramid and Palm Trees Test, Boston Naming Test, Rey-Osterrieth Complex Figure, Reverse Digit Span subtest, Oral Trail Making Test, Edinburgh Cognitive, Behavioural ALS Screen (ECAS), Griffiths scale, Child Behavior Check List (CBCL), Youth Self Report (YSR),Strength and Difficulties Questionnaire (SDQ), Mini Mental State
Examination (MMSE), Addenbrooke's Cognitive Examination-Revised (ACE-R), California Verbal Learning Test (CVLT), Stroop Color and Word Test (SCWT).

Outcome: The primary outcome measure was the potential ability to accurately assess neuromuscular diseases and their implications for cognitive impairment through screening: Genetic analysis, MRI scans, mobile transcutaneous sensor for tissue blood gas tensions and cognitive Scales.

Papers were assessed for eligibility based on title and abstract; 80 were classified as meeting eligibility criteria. The next stage of the winnowing process involved full-text screening of the potentially relevant papers; Eventually, 63 studies were included in the review.

\section{Results}

Seventeen experimental studies and 30 case reports with cognitive scales and neuromuscular assessment using scales, as well as genetic studies and neuroimaging studies with MRI, 16 review papers about neuromuscular diseases. This study demonstrated that the neuromuscular diseases are multifactorial pathologies characterized by extensive muscle fiber damage that leads to the activation of satellite cells and to the exhaustion of their pool, with consequent impairment of the regenerative process.

\section{The prospective population-based study of cognitive impairment in neuromuscular diseases}

The majority of neuromuscular diseases patients presented with features consistent with executive dysfunction, and have shorter survival time. This last finding, in turn, suggests that studies reliant on prevalent cases are likely to underestimate the incidence of these frontotemporal syndromes. In patients without dementia, we observed an overrepresentation of executive dysfunction. The incidence of executive impairment in this cohort was higher using the screening criteria. The cognitive evaluation and cognitive tasks demand heavily on multiple frontostriatal circuits leading to sensitivity in detecting any disturbance of neural circuits established for cognitive and motor demands.

In this present study, neuromuscular diseases patients showed generally a moderate muscular impairment, impaired cognitive performance over a broad range of functions, including frontal, visuospatial, naming and memory abilities and working memory abnormalities. The degree of cognitive impairment in patients with neuro- muscular diseases is greatly variable, and significant cognitive impairment is observed only in some patients. Symptoms such as depression and anxiety as well as deficits of visuospatial abilities, executive functions, and naming have been frequently reported. Several MRI studies have shown that neuromuscular diseases patients have generally more deficiencies of neural connections in the frontal, parietal and temporal regions and; these dysfunctions increase with the progression of the disease.

\section{Discussion}

\section{Motor neuron diseases and cognitive impairment}

Motor Neuron Disease (MND) is a degenerative disease caused by motor neuron degeneration, whether inferior or superior. Amyotrophic Lateral Sclerosis (ALS), the most well-known form of MND, is a devastating severe disease that simultaneously affects the lower motor neuron (LMN) and upper motor neuron (UMN).9,10 Descriptions in the literature have been linking, for more than a century, emotional liability with bulbar-onset ALS. After pathological and genetic discoveries, over the years, the overlap of frontotemporal dementia (FTD) and MND was strengthened. 9,10 Data from recent studies show that more than $50 \%$ of ALS patients at some point in the disease course, will develop phenotypic picture of cognitive or behavioural impairment, and about 13\% will exhibit concomitant behavioural-variant frontotemporal dementia (b-FTD). 10-16 Patients who present cognitive or behavioural changes, that do not fulfil all the formal criteria for FTD, can be grouped into three different categories: ALS with behavioural impairment; ALS with executive dysfunction; and ALS non-executive dysfunction. 10,17,18

Frontotemporal dysfunction, when present, is strongly linked to lower survival in patients with ALS. In addition, regardless of the age of onset, delayed diagnosis, baseline severity, education and respiratory status, the presence of executive dysfunction is a factor and risk for a worse prognosis. ${ }^{10,16}$ Unless FTD is advanced enough to cause significant frontal and temporal lobe atrophy, neuroimaging is rarely useful in diagnosing cognitive and behavioural deficits in ALS. Studies of neural networks have been increasingly used for the analysis of frontotemporal dysfunction in ALS. In studies with resting state functional MRI, it has been observed three main dysfunctional networks in ALS: the salience network, the 
default mode network, and the central executive network. ${ }^{16,19}$ Several screening tools specifically created for rapid neuropsychological assessment of patients with ALS. Some of these tools, currently available, include the ALS-Brief Cognitive Assessment (ALS-BCA), the ALSCognitive Behavioural Screen (ALS-CBS), the ALS-FTD-Q, Edinburgh Cognitive and Behavioural ALS Screen (ECAS). Patients who are in compliance with the cut-off score of these tests have formal indication of complete neuropsychological assessment. 10,20-22

In addition, the GGGGCC hexanucleotide repeat expansion in the chromosome 9 open reading frame 72 (C9orf72) is a frequent cause of familial MND, FTD and especially frontotemporal dementia-motor neuron disease. The repeat expansion of C9orf72 results in dipeptide repeat proteins (DPRs), which form inclusions in central nervous system (CNS). Finally, it induces the mislocalization and aggregation of TAR DNA-binding protein 43 (TDP-43). It is estimated that in the European population, 50 to $70 \%$ of patients with familial frontotemporal dementia-motor neuron disease and 15 to $20 \%$ with apparent sporadic familial frontotemporal dementia-motor neuron disease, exhibit the expansion of the C9orf72 gene. The penetrance of C9orf72 gene expansion has not yet been determined, but it seems unlikely to be a complete penetrance. $18,23,24$ TAR DNA-binding protein of $43 \mathrm{kDa}$ (TDP-43), was proved to be present in neuronal cytoplasmic inclusions and dystrophic neurites in the hippocampal region and temporal cortex of patients with frontotemporal dementiamotor neuron disease.

Although C9orf72 is the most frequent genetic cause of frontotemporal dementiamotor neuron disease, other gene mutations (VCP, SQSTM1, OPTN and UBQLN2) have already been linked to this pathology, but they are rare causes. ${ }^{23}$

Another MND with cognitive impairment is the bulbospinal muscular atrophy or Kennedy's Disease (KD). KD is an Xlinked recessive pathology that affects men and is caused by the repetition of the CAG trinucleotide in exon 1 of the androgen receptor gene, slowly progressive, with adult-onset. It is characterized by degeneration of LMN that leads to atrophy, weakness, and fasciculation of limbs and bulbar musculature. A striking feature in KD is the presence of minipolymyoclonus. ${ }^{25}$ It is required more than 38 repeats to consider the CAG trinucleotide expansion as pathogenic. The CAG repeat size correlates inversely with disease onset, but not with disease progression or severity. ${ }^{25}$ Systemic
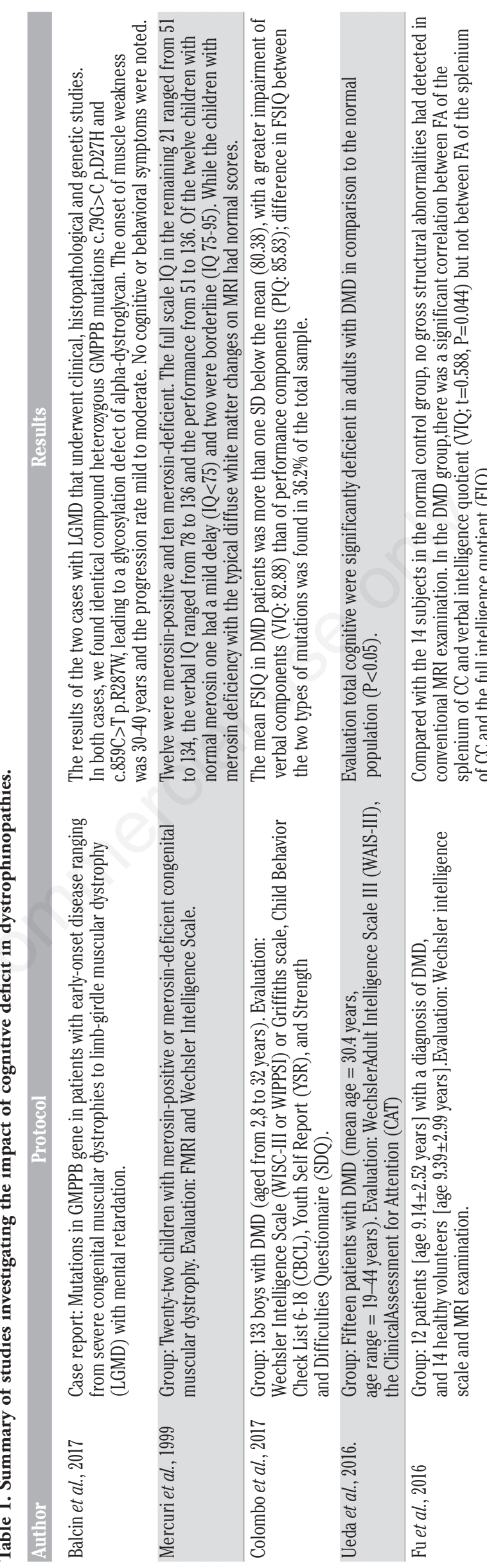
symptoms are also found in $\mathrm{KD}$. The most common non-neurological manifestation is gynecomastia. In addition, reduced fertility due to testicular atrophy, oligo/azoospermia, erectile dysfunction and reduced libido may be present. 25,26 Cognitive impairment may be found in KD. Guidetti et al. ${ }^{26}$ have observed in their patients a predominant impairment in long-term memory and selective attention. Kessler et al. ${ }^{27}$ observed clinical characteristics of frontal lobe dysfunction with disinhibition, impaired executive functions and reduced affective modulation. 27

\section{Cognitive impairment of muscular dystrophies: dystrophinopathies}

Congenital muscular dystrophies (CMDs) are clinically and genetically heterogeneous inherited disorders that compass muscle weakness typically manifested at birth or in infancy. ${ }^{11}$ The spectrum ranges from severe floppy infant syndrome to moderate motor delay and mild or moderate limb-girdle involvement during childhood, compatible with survival into adult life and relatively good quality of life. 6 Besides muscle compromise, white matter and structural abnormalities of the brain, seizures, cognitive impairment, and eye abnormalities may occur depending on the genetic cause. Mutations occur in genes encoding structural proteins of the extracellular matrix, glycosylation enzymes, and proteins of the endoplasmic reticulum (ER) and nuclear envelope. However, just in 25$50 \%$ of CMD-affected subjects, mutations can be identified, suggesting that there are additional genes to be acknowledged. ${ }^{11}$ On the other hand, it is frequently unfeasible to establish a one-to-one relationship between a given gene and a defined phenotype. For example, mutations in the FKRP (Fukutinrelated protein) gene may lead to manifestations extended from the severe WalkerWarburg (WW) syndrome to the late adultonset limb-girdle muscular dystrophy (LGMD). ${ }^{28}$ The genetic defects might not only interfere with the translated sequence, but also with the posttranslational processing, as described with $\alpha$-dystroglycan, a major extracellular matrix receptor on muscle. 28

The term dystroglycanopathies encompass the mutation in the dystroglycan gene (DAG1) itself or mutations in genes encoding glycosyltransferases of alpha-dystroglycan $(\alpha-D G)$, which are necessary to allow $\alpha$ DG binding to laminin. ${ }^{29,30}$ In brain, dystroglycan is expressed in astrocytic end feet abutting the glia limitans and the intracerebral vasculature, in photoreceptor cells of the retina, and in major neurons and glia in the developing CNS. ${ }^{29}$ Cases of milder course of the disease seem to be related to a less severe and remaining glycosylation of $\alpha$-DG. ${ }^{30}$ In the more severe end of the clinical spectrum, there are brain and eyeabnormalities, as illustrated by: Fukuyama congenital muscular dystrophy (FCMD), muscle-eye-brain (MEB) disease and WWS, examples of a continuum in increasing of severity.30,31 The typical CNS involvement among dystroglicanopathies includes various degrees of lissencephaly type II (also known as cobblestone complex), pachygyria, neuronalheterotopias, ponto cerebellar hypoplasia, and cerebellar cysts. ${ }^{28}$

Merosin-deficient congenital muscular dystrophy also referred to as MDC1A, is caused by mutations in the LAMA2 gene, which encodes the $\alpha 2$ subunit of laminin (merosin).29 Patients manifest severe muscular weakness and atrophy, diffuse contractures, inability to walk and facial dysmorphism. ${ }^{31}$ Ventilatory is needed within the first 10 years for most patients. ${ }^{28}$ Since laminin- $\alpha 2$ chain is localized to the basal lamina of all cerebral blood vessels, the lack of $\alpha 2$ may lead to a disruption of the bloodbrain barrier (BBB), increased water content, and a resulting abnormal white matter signal intensity (WM). ${ }^{31}$ The typical abnormal signal in WM seem in T2-wheited MRI (T2 MRI) spares the compacted fibre tracts such as the corpus callosum (CC). The cognitive functions are not impaired if the WM changes are isolated. ${ }^{28}$ Nevertheless, it has been reported a child with cortical dysplasia and severe mental retardation, and a series of children who in addition to the typical findings had cerebellar hypoplasia and a lower performance IQ. ${ }^{32}$

Duchene muscular dystrophy (DMD) is one of the most common genetic disorders, affecting about $1: 3000$ boys. ${ }^{33,34}$ It is an Xlinked dystrophy caused by genetic mutations disrupting the protein dystrophin (DYS), another core protein of dystrophinglycoprotein Complex (DGC), as DG. ${ }^{29,33,34}$ DMD is characterized by progressive muscle degeneration and non-progressive cognitive deficits. $33-35$ DYS also plays an important role in the architectural organization of the CNS. ${ }^{33}$ It is involved in the organization of GABAA receptors, once is normally expressed at the postsynaptic membrane in inhibitory synapses of structures related to cognition and emotional behaviour, such as hippocampus, amygdala, cerebellum and sensory cortices. ${ }^{7,8}$

Although dystrophin expression in brain is only one-tenth of that found in muscle, brain tissue exhibits much greater variability in the protein products from the dystrophin gene. It includes the full-length protein, Dp427, and shorter proteins being
Dp71 the main product expressed in the brain. Dp71 is found around perivascular astrocyte end feet, which suggests its role in BBB function and possible in the possible access of peripheral inflammatory molecules into the brain. In the CNS, Dp427 is only found in neurons, and only within specific regions of the brain such as the hippocampus, amygdala, cerebellar Purkinje cells, and neocortex. The interaction of Dp47 with DGC would be important for the formation and maintenance of new synaptic connections. ${ }^{36}$

The lack of dystrophin in $\mathrm{mdx}$ mice) seems to alter neuron proliferation, survival and/or differentiation and perturb neuronal migration (The density of CA1 pyramidal neurons was reduced by $\sim 34 \%$ in the anterodorsal hippocampus, although quantitative reductions varied along the hippocampal antero-posterior axis. ${ }^{35}$ In their hippocampus were identified cytokines, which are also chronically elevated in peripheral tissue and plasma of patients with DMD. Key proinflammatory cytokines such as IL-1, TNF- $\alpha$, and IL-6 exert neuromodulatory effects on the hippocampus and have been linked to altered capacities for learning and the formation of memories. Hence, there may be a neuroimmune contribution in the CNS disturb of dystrophindeficient patients. ${ }^{36}$

In mice, the loss of DYS leaded to enhanced unconditioned fears, anxiety and deficits in fear conditioning and memories, suggesting the role of the protein in the functioning of the neuronal circuit of fear. ${ }^{7}$ The absence of dystrophin resulted in a significant elevation of $[\mathrm{Ca} 2+] \mathrm{i}$ and $[\mathrm{Na}+] \mathrm{i}$ in intact adult cortical and especially hippocampus pyramidal neurons of mice, as well as an increase in ROS production, and cell death. This ion dyshomeostasis was related to a spatial learning deficit. 34

In humans, the lack of this protein might also lead to neurobehavioral disorders, including attention-deficit/hyperactivity disorder (ADHD), autism spectrum disorders, and obsessive-compulsive disorder. In the context, Colombo et al. ${ }^{33}$ reported a non-progressive lower IQ in DMD boys compared to controls. Boys may present specific learning disabilities such as dyslexia and impaired executive functions, namely problem solving, inhibition and working memory. 33 In addition, Astrea et al. 37 demonstrated that 15 patients with DMD (age range $=19-44$ years) demonstrated impairment in the ability to sequentially process auditory and visual information and attention. ${ }^{6}$ A controlled study of diffusion tensor imaging (DTI) involving 15 boys with DMD showed significantly lower fractional anisotropy (FA) values in the spleni- 
um of CC and these values were positively correlated with verbal IQ. ${ }^{37}$

A recent study assessing 47 Italian boys with DMD found that $14.89 \%$ of the boys had a diagnosis of ASD. Full Scale Intelligence Quotient (FSIQ) $<70$ were registered in $36.2 \%$ of them, with a greater impairment of verbal than other performance components. ${ }^{33}$ Distal DMD mutations were implicated to more severe cognitive deficit, as reported by previous studies.6,33 Similar results were found in a longitudinal study following 41 preschool DMD boys until school age. It was confirmed a correlation between neurodevelopmental assessments and later cognitive scales and on both evaluation the overall results were better when the mutation was upstream or in the exon 44 without affecting Dp140 expression. ${ }^{38}$ Distal mutations are associated with loss of cerebral dystrophin isoforms, which are more expressed in the cerebellum and limbic system. It is worth to remember that literature supports the cerebellar contribution even to aspects of social emotional and regulatory behaviour. ${ }^{33}$

Mutations in the DYS gene are also responsible for causing Becker muscular dystrophy (BMD). However, in BMD there is a production of partially functional dystrophin. Patients typically remain ambulatory at least until age 15 and commonly well into adult life. Cognitive impairment and neurobehavioral comorbidities are also not as common or severe in BMD compared with DMD. 39

Myotonic dystrophies (DM) are autosomal dominant multisystemic disorders that affect also the brain. 40 Myotonic dystrophy type 1 (DM1) is caused by a CTG trinucleotide repeat expansion in the non-coding region of the dystrophiamyotonica-protein kinase $(D M P K)$ gene, resulting in alternative splicing of pre-messenger RNAs for various proteins, including the TAU and NMDA.6,40 Cognitive deficits have been observed in $24-75 \%$ of DM1 patients and it depends on the age at onset of the disease.6,31,40 Congenital patients tend to have mental retardation, while those with onset in the childhood have learning disabilities due to lower IQ, the ADHD, autistic behaviour, visuospatial impairment, lack of interest and inhibition. Adult onset is related to the dysexecutive syndrome and visuospatial deficit, while verbal functions and memory seem to be age dependent. Lateonset DM1 patients were reported to have predominantly age-dependent memory deficit. 40

Lower IQs seems to be correlated with longer expansions, mainly related to maternal inheritance and age of onset of symptoms although it does not represent a more severe neuromuscular impairment. ${ }^{6}$ Neuropsychological analysis of 101 DM1 patients (childhood, juvenile, adult, and late-onset) registered normal general intellectual level. Visuospatial impairment was the main cognitive defect with prevalence of $75-86 \%$ among different phenotypes. Executive dysfunction was the second most common cognitive impairment, affecting $60-79 \%$ of the sample. Adult DM1 subjects with older age and longer duration of disease were more susceptible to memory and language defects. Late onset was associated with the affection of multiple cognitive domains including the highest prevalence of memory impairment (more than 80\%). Among 46 DM2 patients, it was showed that even one-third of them had completely normal neuropsychological findings. Visuospatial and executive dysfunctions were also the most prevalent in DM2, but less common than in DM1.40

A longitudinal neuropsychological assessment among 115 DM1 - only adult phenotypes disclosed that executive functions (Stroop color-word, fluency tests and WAIS-R for digit symbol coding and block design), language (fluency and Boston Naming Test), and visual memory (Rey Complex Figure Test) were the most impaired cognitive functions at baseline. However, they did not significantly decline through the time, in opposition to verbal memory, processing speed, and visual attention, which, actually, also imply the requirement of some specific executive functions (i.e. processing speed itself, mental flexibility, and selection and maintaining of accurate strategies). The FSIQ and the visual construction also remained stable. The adult phenotype performed more poorly than the late-onset phenotype on most of the tests, both at baseline and follow-up. Nevertheless, the rate of decline was higher in the late-onset than in the adult phenotype. The level of progression in most of the cognitive performances correlated with age and disease duration but not with the CTG repeats, educational level nor muscular impairment. These findings are consistent with the hypothesis that DM1 could be considered as a progeroid disease (an accelerated and increased ageing process). ${ }^{41}$

A comparative study involving nine DM1 patients and age matched healthy controls using T1-weighted and DTI disclosed changes in cortical thickness and WM in the widely distributed network of the frontal, temporo-occipital, medial parietal, and sensorimotor areas. CTG repeats were negatively correlated to thickness in the right medial orbitofrontal cortex, left middle temporal, supramarginal, and the lateral occipital cortex and to the WM integrity in the left entorhinal, anterior corona radiate, orbitofrontal, and lateral occipital areas. This study revealed higher occurrence of WM than cortical abnormalities. This finding together with the correlation between WM lesion severity and disease duration suggests that DM1 represents a slow demyelinating process, which eventually progresses to axonopathy. Moreover, the decreased integrity of connectivity, as well as cortical pathology, was located mostly in the ventral side connecting the fronto-temporal cortex. These findings may be relevant to the behavioural symptoms, including dysexecutive syndrome, apathy, and depression. In that study, diffusion metrics were also affected in the external capsule, which might be related to decrease verbal fluency resulting from impairment in semantic processing. ${ }^{42}$ Bajrami et al. ${ }^{43}$ suggest that the WM hyperintense lesions (WMHLs) located at the temporal poles seem to be characteristics of the disease, while others, small and diffuse WMHLs would be similar to the age-related alterations. 43

\section{Mitochondrial disorders and cogni- tive impairment}

Mitochondrial diseases (mtD) are those caused by mutations in mitochondrial DNA (mtDNA) and/or nuclear DNA (nDNA) genes due the dual genetic control (nDNA and mtDNA) of the respiratory chain (RC), the complexity of intergenomic signalling and its functional consequences. ${ }^{44,45}$ The mutation rate for mtDNA is much higher than that for nuclear DNA, especially for deletions. ${ }^{46}$ The diagnosis of $\mathrm{mtD}$ is challenging since the clinical picture is varied and can occur at almost any stage of life, often with involvement of unusual combination of organs. ${ }^{45,47}$ Systems/organs most frequently clinically or subclinically affected in $\mathrm{mtD}$ are the peripheral nervous system (PNS), the central nervous system (CNS), endocrine glands, heart, ears, eyes, gastrointestinal tract, liver, kidneys, bone marrow, and dermi. The second most frequently affected system is the CNS.48 Common CNS findings are fluctuating encephalopathy, seizures, migraine, stroke-like episodes, ataxia, spasticity and cognitive decline through dementia. 49 The cognitive decline among manifestations of CNS is increasingly recognized and diagnosed, it is essential to guide the optimal management of these patients.

Mitochondrial syndromes associated with cognitive impairment due to mtDNA mutations include mitochondrial encephalopathy, lactic acidosis and strokelike episodes (MELAS) syndrome, myoclonus epilepsy with ragged-red fibres 
(MERRF) syndrome, maternally inherited diabetes and deafness (MIDD), mitochondrial chronic progressive external ophthalmoplegia (CPEO), Kearns-Sayre syndrome (KSS) and neuropathy, ataxia and retinitis pigments (NARP) syndrome. ${ }^{50}$ The assessment of cognitive functions in these patients is made through a neuropsychological test, and the domains impaired were abstract reasoning, verbal memory, visual memory, language, executive or constructive functions, calculation, attention or visuospacial functions. Cognitive functions and intellectual abilities may decline from initially focal cognitive impairment to lastly dementia. ${ }^{51}$ In addition, the investigation proposed to detect mitochondrial dementia is based on imaging studies, including MRI, PET, SPECT or MR-spectroscopy, cerebrospinal fluid (CSF) investigations or electroencephalography. 52

The mitochondrial disorders are a genetically heterogeneous group and variable phenotype, therefore we select the most frequent mitochondrial diseases with cognitive impairment to approach:

MELAS - mitochondrial encephalopathy, lactic acidosis and stroke-like syndrome is typified by characteristic neurological manifestations including seizures, encephalopathy, and stroke-like episodes, and other secondary manifestations just like short stature, cognitive impairment, migraines, depression, cardiomyopathy, cardiac conduction defects and diabetes mellitus. ${ }^{53}$ The patients suffer from cognitive dysfunction of nuclear pathogenesis. Emmanuele et al. ${ }^{54}$ present a study exploring a possible link between cognitive dysfunction and hippocampal expression of calbindin D 28KD (CB), a high-affinity calcium-binding protein, in four MELAS patients, using post mortem hippocampal tissues. Reduction in CB expression is associated with aging and with neurodegenerative disorders. They found a reduction in $\mathrm{CB}$ expression in these patients and postulated that the reduced $\mathrm{CB}$ expression may play a role in the cognitive abnormalities in MELAS. ${ }^{54}$

MERRF - Myoclonus Epilepsy associated with Ragged-Red Fibers had as diagnostic criteria typical manifestations of the disease such as myoclonus, generalized epilepsy, cerebellar ataxia and ragged-red fibres on muscle biopsy. Other multisystemic alterations can be found as: deafness, exercise intolerance, peripheral neuropathy, abnormal cardiac conduction, dementia, among others. ${ }^{55}$ Kaufmann et al. ${ }^{56}$ studied 91 individuals from 34 families with MELAS and the A3242G point mutation and 15 individuals from two families with MEERF and the A8344G mutation. The patients were divided into groups (asymptomatic, oligosymptomatic and symptomatic), and submitted to neurologic examination, neuropsychological testing and spectral resonances. The study showed that the MELAS probands had higher ventricular lactate estimates on spectral resonances than the MEERF probands, and the MELAS probands had lower scores on formal neuropsychological testing than MEERF probands. In this way the authors correlated high levels of ventricular lactate, the brain spectroscopic signature of MELAS are associated with more severe neurologic impairment. The study also evidenced that the 15 patients with MEERF had score for degree of impairment on neurologic examination lower than MELAS patients and that worsening of neuropsychological functions was independent of the severity of clinical manifestations. 56

Kearns-Sayre syndrome (KSS) presents that classic triad: pigmentary retinopathy, chronic progressive external ophthalmoplegia and heart block, beyond features like cerebellar ataxia.57 Chronic Progressive External Ophthalmoplegia (CPEO) is a mitochondrial myopathy with slowly progressive, often symmetric blepharoptosis and limitation of ocular motility in all directions of gaze. 58 Bosbach et al. ${ }^{59}$ performed a study with 22 patients with CPEO or KSS included screening for mitochondrial DNA (mtDNA) point mutations (3243/8344) and mtDNA deletions. All patients underwent a neuropsychological test battery. The neuropsychological testing did not reveal signs that would suggest general intellectual decline or dementia, but provided evidence of specific focal neuropsychological deficits, suggesting particular impairment of visuospatial perception associated to parieto-occipital lobes and executive deficits associated to the prefrontal cortex. 59

Autosomal CPEO presents with similar manifestations as mitochondrial CPEO but is due to mutations in the twinkle, POLG1 or ANT1 genes. ${ }^{50}$ Simoncini et al. 60 reported a case of a woman with ANT1- related adPEO and dementia. The patient started to report disorders of short-term memory, and the neuropsychological evaluation showed a mild impairment of verbal memory and the attentive functions, magnetic resonance of brain revealed widespread moderate cortical atrophy, and magnetic resonance spectroscopy of brain disclosed mild elevation of lactate in the posterior interhemispheric region and in ventricular region. However, studies are needed to assess the prevalence of central neurological manifestations in ANT1 mitochondrial disease. ${ }^{60}$

Leigh syndrome also referred to as subacute necrotizing encephalomyelopathy is a

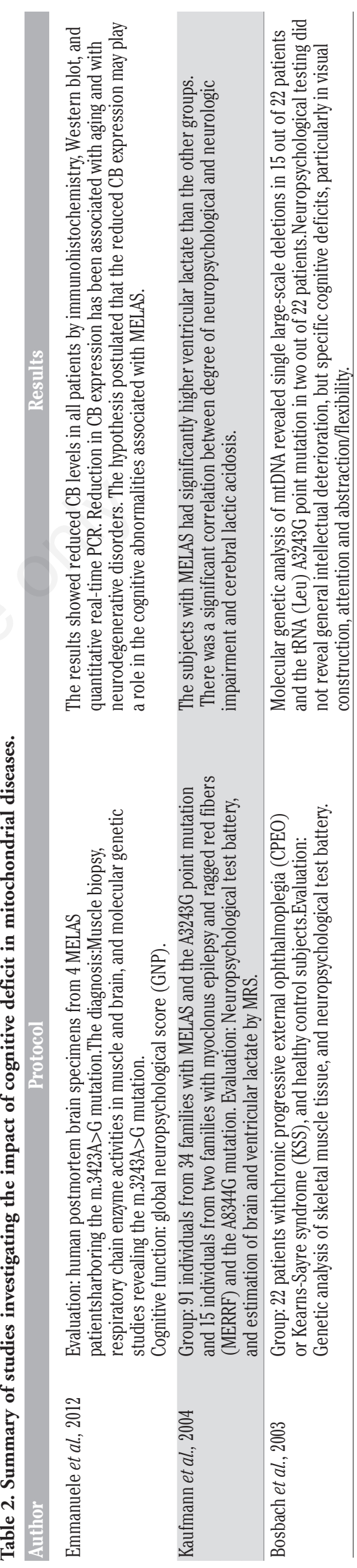




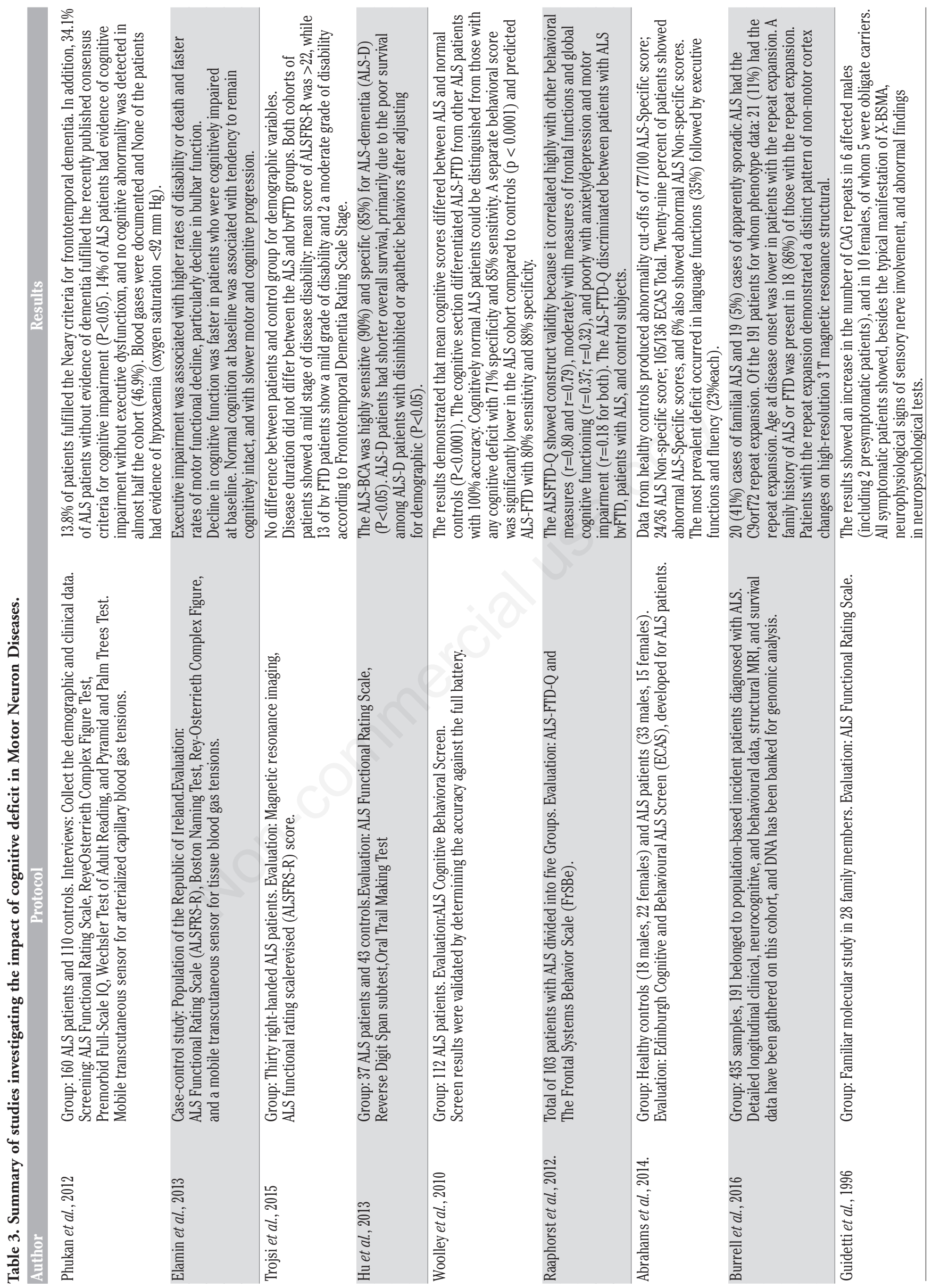


disease that results in the regression of both mental and motor skills, leading to disability and rapid progression to death. ${ }^{61}$ The patients also present refractory seizures, optic atrophy, ophthalmoparesis. Mental development is typically delayed at early age. Imaging studies show focal, bilaterally symmetric lesions of the basal ganglia, the thalamus and/or the brainstem. Moreover, there is raised lactate levels in blood and/or cerebrospinal fluid. 50,61

Mitochondrial neurogastroinstestinal encephalomyopathy (MNGIE) is a rare autosomal recessive disorder in which the patients suffer from gastrointestinal dysmotility, cachexia, ptosis, external ophthalmoparesis, myopathy, and polyneuropathy. MRI shows leukoencephalopathy. ${ }^{62}$ CarodArtal et al. ${ }^{63}$ reported a case of a Brazilian man with MNGIE who had poor weight gain, muscular atrophy, and chronic diarrhoea since childhood. He presents episodic gastric pain, recurrent nausea and vomiting, muscle aching and cramps, and had signs of memory impairment. On neuropsychological examination, patient scored $28 / 30$ on MMSE. Working memory scores were $14 / 25$ and $7 / 25$ on immediate recall, and $6 / 25$ and $1 / 25$ on long-term recall. The patient could repeat seven digits in direct order and only four digits in reverse sequence. Verbal learning was involved (7/15 words). 63

The treatment of mitochondrial dementia includes general measures and specific therapy, which encompass: physiotherapy and physical exercise below the maximal individual limit; dietary measures; avoidance of mental and physical stress, alcohol, nicotine and infections; supplementation of respiratory chain components such as coenzyme Q., Various studies have reported a positive effect of coenzyme $Q$ in mitochondrial dementia; administration of artificial electron acceptors, such as vitamin $\mathrm{C}$ or $\mathrm{K}$; administration of metabolites or cofactors like carnitine. Overall, cognitive decline in mitochondrial dementia needs to be included in the differentials of dementia. 52

\section{Limitations of existing research}

There are limitations to our study. In terms of research strategy and data collection, we opted to evaluate only studies that we found through the ISI Web of Science and PubMed database, which may have limited the number of included studies. Furthermore, we only looked at studies written in English. Concerns about the reliability and validity of the applicability conditions of the questionnaires, as well as their potential subjectivity of neurofunctional and cognitive evaluation, to biased estimates of intervention effectiveness. Another potential limitation is the possible confounding variables in case reports and the low sample size.

The majority of the findings are based cross-sectional methods; further longitudinal studies are needed in order to model within-person change and avoid cohort effects, have been conducted. There are also other sources of variability within the literature that may have limited the conclusions drawn from this review, such as the lack of standardization in the cognitive and neuromuscular evaluation. This plethora of effects poses a serious challenge to researchers seeking neuromuscular diseases unifying principles. Limitations were also secondary to population size and phenotypes in relation to sample size in genetic research. In addition, the studies differ in terms of neuroimaging analysis.

\section{Conclusions}

The literature about cognition in neuro muscular diseases is still scarce, but studies converge on the influence of these disorders on mental processes (see Tables 1-3 for a summary on previous studies). Congenital disorders are common to neuromuscular function and brain function impaired. Considering that severe neuromuscular conditions such as DMD and ALS have shown a marked improvement in prognosis in the last decades, the search for the improvement of patients' quality of life has become increasingly important, which can be compromised by the primary and secondary cognitive effects the evolution of the disease. More work should be done to better evaluate cognitive deficits in patients with neuromuscular disorders, in order to improve the patient's relationship with the medical staff, the family, and society and improve the patient's quality of life. In addition, knowing the evolution of the cognitive symptoms of each disease is important for proper management of the patient, evaluation of survival, establishment of prognosis and choice of appropriate therapeutic intervention.

\section{References}

1. Katz JA, Murphy GS. Anesthetic consideration for neuromuscular diseases. Curr Opin Anaesthesiol 2017;30:1.

2. Papapetropoulos S, Ellul J, Argyriou AA, et al. The effect of depression on motor function and disease severity of Parkinson's disease. Clin Neurol
Neurosurg 2006;108:465-9.

3. Nieuwenhuys A, Oudejans RRD. Anxiety and perceptual-motor performance: toward an integrated model of concepts, mechanisms, and processes. Psychol Res 2012;76:747-59.

4. Krane-Gartiser K, Steinan MK, Langsrud $\mathrm{K}$, et al. Mood and motor activity in euthymic bipolar disorder with sleep disturbance. J Affect Disord 2016;202:23-31.

5. Argento AG, Kaufman J. Cognition in neuromuscular disease. J Pediatr Rehabil Med 2008;1:199-210.

6. Astrea G, Battini R, Lenzi S, et al. Learning disabilities in neuromuscular disorders: a springboard for adult life. Acta Myol myopathies cardiomyopathies Off J Mediterr Soc Myol 2016;35:90-5.

7. Vaillend C, Chaussenot R. Relationships linking emotional, motor, cognitive and GABAergic dysfunctions in dystrophin-deficient mdx mice. Hum Mol Genet 2017;26:1041-55.

8. Ueda Y, Suwazono S, Maedo S, Higuchi I. Profile of cognitive function in adults with duchenne muscular dystrophy. Brain Dev 2016;1-6.

9. Mitchell JD, Borasio GD. Amyotrophic lateral sclerosis. Lancet. 2007;36:203141.

10. van Es MA, Hardiman O, Chio A, et al. Amyotrophic lateral sclerosis. Lancet 2017;6736:1-15.

11. Wiessner M, Roos A, Munn CJ, et al. Mutations in INPP5K, Encoding a Phosphoinositide 5-Phosphatase, Cause Congenital Muscular Dystrophy with Cataracts and Mild Cognitive Impairment. Am J Hum Genet 2017; 100:523-36.

12 Phukan J, Elamin M, Bede P, et al. The syndrome of cognitive impairment in amyotrophic lateral sclerosis: a population-based study. J Neurol Neurosurg Psychiatry 2012;83:102-8.

13. D’Angelo MG, Bresolin N. Cognitive impairment in neuromuscular disorders. Muscle Nerve 2006;34:16-33.

14. Moher D, Liberati A, PRISMA Group. Preferred Reporting Items for Systematic Reviews and MetaAnalyses: The PRISMA Statement. Ann Intern Med 2009;151:264-9.

15. Elamin M, Bede P, Byrne S, et al. Cognitive changes predict functional decline in ALS: A population-based longitudinal study. Neurology 2013;80:1590-7.

16. Woolley SC, Strong MJ. Frontotemporal Dysfunction and Dementia in Amyotrophic Lateral Sclerosis. Neurol Clin 2015;33:787- 
805.

17. Strong MJ, Grace GM, Freedman M, et al. Consensus criteria for the diagnosis of frontotemporal cognitive and behavioural syndromes in amyotrophic lateral sclerosis. Amyotroph Lateral Scle. 2009;10:131-46.

18. Byrne S, Elamin M, Bede $\mathrm{P}$, et al. Cognitive and clinical characteristics of patients with amyotrophic lateral sclerosis carrying a C9orf72 repeat expansion: a population-based cohort study. Lancet Neurol 2012;11:232-40.

19. Trojsi F, Esposito F, de Stefano M, et al. Functional overlap and divergence between ALS and bvFTD. Neurobiol Aging 2015;36:413-23.

20. Hu WT, Shelnutt M, Wilson A, et al. Behavior Matters-Cognitive Predictors of Survival in Amyotrophic Lateral Sclerosis. PLoS One 2013;8.

21. Woolley SC, York MK, Moore DH, et al. Detecting frontotemporal dysfunction in ALS: Utility of the ALS Cognitive Behavioral Screen (ALSCBS (TM)). Amyotroph Lateral Scler 2010;11:303-11.

22. Raaphorst J, Beeldman E, Schmand B, et al. The ALS-FTD-Q: A new screening tool for behavioral disturbances in ALS. Neurology 2012;79:1377-83.

23. Burrell J, Halliday G, Kril J, et al. The frontotemporal dementia - motor neuron disease continuum Popular Articles. Front Dementia Motor Neuron Dis Contin 2016;388:919-32.

24. Orsini M, Ferreira ACADF, Nascimento OJM, et al. Frontotemporal dementia in amyotrophic lateral sclerosis: From rarity to reality? Neurol Int 2016;8:33-5.

25. Querin G, Sorarù G, Pradat PF. Kennedy disease (X-linked recessive bulbospinal neuronopathy): A comprehensive review from pathophysiology to therapy. Rev Neurol (Paris) 2017; 173:326-37.

26. Guidetti D, Vescovini E, Motti L, et al. $\mathrm{X}$-linked bulbar and spinal muscular atrophy, or Kennedy disease: clinical, neurophysiological, neuropathological, neuropsychological andmolecular study of a large family. Clin Neurophysiol 1996;135:140-8.

27. Kessler H, Prudlo J, Kraft S, Supprian T. Dementia of frontal lobe type in Kennedy's disease. Amyotroph Lat Scler Mot Neuron Disord 2005;6:250-3.

28. Schessl J, Zou Y, Bönnemann CG. Congenital Muscular Dystrophies and the Extracellular Matrix. Semin Pediatr Neurol 2006;13:80-9.

29. Zhang QZ. Dystroglycan induced muscular dystrophies - a review. Eur Rev Med Pharmacol Sci 2016;20:3683-7.
30. Balcin H, Palmio J, Penttilä S, et al. Late-onset limb-girdle muscular dystrophy caused by GMPPB mutations. Neuromuscul Disord 2017;27:627-30.

31. Reed UC. Congenital muscular dystrophy - Part II: A review of pathogenesis and therapeutic perspectives. Arq Neuropsiquiatr 2009;67:343-62.

32. Mercuri E, Gruter-Andrew J, Philpot J, et al. Cognitive abilities in children with congenital muscular dystrophy: correlation with brain MRI and merosin status. Neuromuscul Disord 1999;9:383-7.

33. Colombo P, Nobile M, Tesei A, et al. Assessingmental health in boys with Duchenne muscular dystrophy: Emotional, behavioural and neurodevelopmental profile in an Italian clinical sample. Eur J Paediatr Neurol 2017; 21:639-47.

34. Lopez JR, Kolster J, Uryash A, et al. Dysregulation of Intracellular $\mathrm{Ca} 2+$ in Dystrophic Cortical and Hippocampal Neurons. Mol Neurobiol 2016;1-16.

35. Miranda R, Laroche S, Vaillend C. Reduced neuronal density in the CA1 anterodorsal hippocampus of the $\mathrm{mdx}$ mouse. Neuromuscul Disord 2016;26:775-81.

36. Rae MG, O’Malley D. Cognitive dysfunction in Duchenne Muscular Dystrophy: a possible role for neuromodulatory immune molecules. J Neurophysiol 2016;2016.

37. Fu Y, Dong Y, Zhang C, et al. Diffusion tensor imaging study in Duchenne muscular dystrophy. Ann Transl Med 2016;4:109.

38. Chieffo D, Brogna C, Berardinelli A, et al. Early neurodevelopmental findings predict school age cognitive abilities in duchenne muscular dystrophy: A longitudinal study. PLoS One 2015;10:1-7.

39. Banihani R, Baskin B, Halliday W, et al. A Novel Mutation in DMD (c. 10797+5G $>$ A) Causes Becker Muscular Dystrophy Associated with Intellectual Disability. J Dev Behav Pediatr 2016;37:239-44.

40. Peric S, Rakocevic Stojanovic V, Mandic Stojmenovic G, et al. Clusters of cognitive impairment among different phenotypes of myotonic dystrophy type 1 and type 2. Neurol Sci 2017;38:415-23.

41. Gallais B, Gagnon C, Mathieu J, Richer L. Cognitive decline over time in adultswith myotonic dystrophy type 1 : A 9-year longitudinal study. Neuromuscul Disord 2017;27:61-72.

42. Yoo WK, Park YG, Choi YC, Kim SM. Cortical thickness and white matter integrity are associated with CTG expansion size in myotonic dystrophy type I. Yonsei Med J 2017;58:807-15.

43. Bajrami A, Azman F, Yayla V, et al. MRI findings and cognitive functions in a small cohort of myotonic dystrophy type 1: Retrospective analyses. Neuroradiol J 2016;30:23-7.

44. Galán F, de Lavera I, Cotán D, SánchezAlcázar JA. Mitochondrial Myopathy in Follow-up of a Patient With Chronic Fatigue Syndrome. J Investig Med High Impact Case Rep 2016;3.

45. Phadke R. Myopathology of Adult and Paediatric Mitochondrial Diseases. J Clin Med 2017;6:64.

46. Jameson E, Morris A. Mitochondrial disease - a review. Paediatr Child Health (Oxford) 2011;21:80-3.

47. McFarland R, Taylor RW, Turnbull DM. A neurological perspective on mitochondrial disease. Lancet Neurol 2010;9:829-40.

48. Finsterer J. Central nervous system manifestations of mitochondrial disorders. Acta Neurol Scand 2006;114:21738.

49. Chinnery PFP. Mitochondrial Disorders Overview. Gene Rev 2014.

50. Finsterer J. Cognitive dysfunction in mitochondrial disorders. Acta Neurol Scand 2012;126:1-11.

51. Finsterer J. Mitochondrial disorders, cognitive impairment and dementia. J Neurol Sci 2009;283:143-8.

52. Finsterer J. Cognitive decline as a manifestation of mitochondrial disorders (mitochondrial dementia). J Neurol Sci 2008;272:20-33.

53. Sproule DM, Kaufmann P. Mitochondrial encephalopathy, lactic acidosis, and strokelike episodes: Basic concepts, clinical phenotype, and therapeutic management of MELAS syndrome. Ann NY Acad Sci 2008; 1142:133-58.

54. Emmanuele V, Garcia-Cazorla A, Huang $\mathrm{H}$ Bin, et al. Decreased hippocampal expression of calbindin D28K and cognitive impairment in MELAS. J Neurol Sci 2012;317:29-34.

55. Lorenzoni PJ, Scola RH, Kay CSK, et al. When should MERRF (myoclonus epilepsy associated with ragged-red fibers) be the diagnosis? Arq Neuropsiquiatr 2014;72:803-11.

56. Kaufmann P, Shungu DC, Sano MC, et al. Cerebral lactic acidosis correlates with neurological impairment in MELAS. Neurology 2004;62:1297302.

57. Ortiz A, Arias J, Cárdenas $\mathrm{P}$, et al. Macular findings in Spectral Domain Optical Coherence Tomography and OCT Angiography in a patient with Kearns-Sayre syndrome. Int J Retin 
Vitr 2017;3:24.

58. Murdock J, Thyparampil PJ, Yen MT. Late-Onset Development of Eyelid Ptosis in Chronic Progressive External Ophthalmoplegia: A 30-Year Followup. Neuro-Ophthalmol 2016;40:44-6.

59. Bosbach S, Kornblum C, Schröder R, Wagner M. Executive and visuospatial deficits in patients with chronic progressive external ophthalmoplegia and Kearns-Sayre Syndrome. Brain 2003;126:1231-40
60. Simoncini C, Siciliano G, Tognoni G, Mancuso M. Mitochondrial ANT-1 related adPEO leading to cognitive impairment: is there a link ? Acta Myol 2017;25-7.

61. Saneto R, Ruhoy I. The genetics of Leigh syndrome and its implications for clinical practice and risk management. Appl Clin Genet 2014;7:221-34.

62. Schüpbach WMM, Vadday KM, Schaller A, et al. Mitochondrial neurogastrointestinal encephalomyopathy in three siblings: Clinical, genetic and neuroradiological features. J Neurol 2007;254:146-53.

63. Carod-Artal FJ, Herrero MD, Lara MC, et al. Cognitive dysfunction and hypogonadotrophic hypogonadism in a Brazilian patient with mitochondrial neurogastrointestinal encephalomyopathy and a novel ECGF1 mutation. Eur J Neurol 2007;14:581-5. 